$\underline{\text { Editorial }}$

\title{
Small is beautiful, but, NANO is incredible
}

\author{
Sri Lanka Journal of Child Health, 2004; 34:72-4
}

(Key words: nanomedicine, nanorobots)

Over many centuries, medicine has progressed through cornerstones of innovations and revolutionary ideas. Long years ago assessment of illnesses was based on the macroscopic appearance of the patient or the organs. Then came the era of micro medicine which heralded unprecedented growth and advancement. However, despite spectacular progress, the state of the current medical scenario has been limited both by a certain lack of understanding and by the tools used. In many ways, medicine has remained as an art, rather than a science ${ }^{1}$.

The technology required to truly understand and repair the human body is molecular technology. After all, the human body is an extremely complex system of interacting molecules or in other words, a molecular machine ${ }^{1}$. Only over the last 50 years or so, did medical science venture into a path leading to examination and treatment of disease pathology at a molecular level. However, from a molecular point of view, despite many impressive advances in the years gone by, medicine has remained rather simple and unsophisticated. Molecular Medicine will necessarily have to "catch up" with the current level of molecular technology in which other fields have rapidly progressed.

Imagine bones woven with a fabric so versatile that one could fall out of a high-rise building but yet walk away unharmed or medical nanites which are capable of consuming atherosclerotic plaques in blood vessels or be able to repair cell damage caused by cancer and tiny machines in the blood stream acting as security guards attacking any foreign entity that enters the bloodstream $^{1}$. What if we could search out and destroy the very first cancer cells that would otherwise have caused a tumour to develop or replace broken parts of cells replaced or could implant pumps the size of molecules to deliver life-saving drugs precisely where and when they are required? May be, these sound like extracts from a narrative on science fiction. Perhaps not quite.., these are real possibilities in the future. Welcome to the world of nanotechnology and nanomedicine.
Small has always been thought to be beautiful and nano means one billionth of a metre or 10-9 metres. Nanotechnology, also called molecular manufacturing, is a branch of engineering that deals with the design and manufacture of extremely small electronic circuits and devices built at the molecular level $^{2}$. Nanomedicine is the application of nanotechnology to monitor, construct and control the human biological system. In simpler words, it means a virtually fool-proof system of prevention and treatment of human disease. A natural consequence of our achieving this level of technology will be the ability to analyse, maintain and repair the human body as completely and as effectively as we can deal with any other machine today. In 1997, a group of health scientists in the United States concluded that if a breakthrough to a molecular assembler occurred within the next 10-15 years, an entirely new field of nanomedicine would emerge by 2020 . They postulated that the initial applications would be outside the human body, in diagnostics and pharmaceutical manufacturing but the most powerful uses would be within the body ${ }^{1}$. They discussed some applications like cell-herding machines to stimulate rapid healing, tissue reconstruction and cell repair machines to perform genetic surgery. Nanomedicine is likely to have extraordinary and far-reaching implications for the medical profession, for the definition of disease, for the diagnosis and treatment of many and varied diseases of the human body and ultimately, for the improvement and extension of natural human biological structure and function ${ }^{3}$.

There are several challenges and problems that have to be overcome in nanotechnological procedures. One such difficulty is the assembly of the actual item in question. Atoms and molecules are not stationary and are constantly moving, jiggling, combining, reacting and ready to confound the would-be molecular architect. Strings of atoms or molecules have to be added to the existing structure to produce the desired effect. Doing this without disturbing the molecular structure of the original structure is no mean task. The position of each atom is specified in a three dimensional format and it becomes a 3-dimensional problem. As atoms and molecules are handled one at 
a time, assembly work has to progress very fast and such speeds are likely to generate mistakes. Thus, together with the assembling process, mistakes have to be detected and corrected. Very large amounts of the product would ultimately be required for a given purpose and at present there are no suitable means of such huge mass production.

A nanomedical company with successful products would collect huge profits. However, they will incur an even higher cost of bringing a product to the market when compared to the present day biotechnology and pharmaceutical companies. It would also be necessary to harness the expertise in many other fields like computer technology, nanopropulsion technology, nanocircuitary etc., to produce the final product.

The problems with drug discoveries today are that it is partly science, partly art and partly luck. The average time to discover and obtain approval for a drug is about 15 years. Even with this, 3 out of 10 new drugs fail the final human testing procedures. This long process plus the protection afforded by patents, allow a relatively long product life cycle for the newer drugs. However, nanomedical products, though protected by patents, will enjoy a much shorter product life cycle. This is simply because things are not left to chance or luck in nanomedicine and every molecule is purpose designed. Once the technology is freely available, the differentiator between companies becomes a function of engineering skills.

There are other minutiae of nanomedicine such as issues on sole proprietorship in view of the collaboration of many different industries in the production of nanoproducts and legal positions in respect of classifying the product as a drug or a device.

One interesting nanomedical application is using deoxyribonucleic acid (DNA) as a construction medium. DNA is thought of as the building blocks of life and the chemical basis of heredity. It is normally not thought of as a building material. Chemists at New York University have developed a means of utilising strands of DNA to construct three dimensional geometric structures. Utilising specific base pairings as the glue, they have linked strands of DNA and introduced bends, kinks, twists etc., which serve as building blocks for even more complex structures. These structures are easily programmable, their arbitrary sequences prevent the body from recognising and utilising them for protein production and they can be modified by a large battery of enzymes. The best use of this expertise is in the de- velopment of drug delivery technology. Such systems are expected to be user-friendly, pleasant, painless, convenient, ultra-efficient and be able to virtually eliminate side effects by targeted drug delivery.

However, the most fascinating application of nanotechnology in medicine is in nanorobotics. Nanorobots are nanodevices that will be used for the purpose of maintaining and protecting the human body against pathogens ${ }^{4}$. They will have a diameter of about 0.5 to 3 microns and will be constructed out of parts with dimensions in the range of I to 100 nanometers. The main element used will be carbon in the form of diamond or fullerene nano-composites which are expected to provide strength and chemical inertness to these forms. Many other light elements such as oxygen and nitrogen can be used for special purposes. To avoid being attacked by the host's immune system, the best choice for the exterior coating is a passive diamond coating. Smoother and more flawless the coating, the less is the reaction from the body's immune system. These nanorobots could be used as programmable antibodies. As disease causing organisms mutate in their endless attempts to get around medical treatments, nanorobots could be reprogrammed to selectively seek out and destroy them ${ }^{2}$.

One nanorobotic discovery that is likely to have a tremendous impact in medicine is the mechanical artificial red cell or the respirocyte ${ }^{5}$. It is a spherical nanomedical device, one micron in diameter, made up of a flawless diamond or sapphire shell and constructed atom by atom. The device is simply an artificial oxygen carrier and utilises an active means of conveying gas molecules into and out of pressurised microvessels. Conventional red cell substitutes have a very short survival time, are not specifically designed to regulate $\mathrm{CO}_{2}$ or to participate in acid-base buffering and several of them produce undesirable vasoconstriction. The radical innovation associated with the respirocyte overcomes many such problems of the conventional red cell substitutes.

Nanomedicine has the potential to virtually eliminate all common diseases of the 20th century and allow extension of human capabilities ${ }^{5}$. The attractive possibilities of this technology in paediatrics, which is plagued by a plethora of infective and genetic diseases, is perhaps endless. May be, just maybe, the most important long term benefit of nanotechnology to human society as a whole could be the dawning of a new era of peace. After all, we could just hope that, people who are independently well-fed, well educated, happy and most of all, disgustingly healthy, would have little motivation to make war ${ }^{5}$. 
As long ago as 1959, acclaimed physicist and Nobel laureate. Richard Feynman suggested that nature could be manipulated at a nanometre scale, atom by atom $^{6}$. Today, although this discipline is still in its infancy, it has the potential to change medical science dramatically in the 21 st century. We are rapidly approaching that era when such manipulations would produce spectacular advances in the diagnosis and treatment of human disease.

\section{References}

1. Tsai Albert. Nanomedicine - The Medical Revolution. Available from:

http://www.usc.edu/org/techalliance/Anthology \%202002/Nanomedi-cine.pdf

2. WHATIS.com: Nanotechnology - searchSMB. com Definitions. Available from:

http://searchsmb.techtarget.com/sDefinition/0, si d44_gci213444,00.html
3. Nanomedicine Book Site. About Nanomedicine (the field). Available from:

http://www.nano-medicine.com

4. Amit Bhargava NANOROBOTS: MEDICiNF OF THE FUTURE. Available from:

http:www.ewh.ieee.org/rlO/bombay/news3 page4 $\underline{\text { html }}$

5. Freitas Robert A. Jr. Nanomedicine FAQ. Available from:

http://www.foresight.org/Nanornedicine/NanoM $\underline{\text { edFAQ.html }}$

6. Forrest D. Room at the bottom. Available from: http://www.innovationwatch.comconnections.2001.03.00.htm

\section{B J C Perera}

Joint Editor 